\title{
Incidence of Early and Late Leaf spots Disease and Agronomic Performance of some Selected Groundnut (Arachis hypogaea L.) Cultivars in Makurdi, Benue State of Nigeria
}

Richard Isa Bwala $^{1 *}$, Zuwaira Haruna Mohammed ${ }^{1}$, Usman Muhammad Maina ${ }^{1}$, Shariifah Saidu Gaya ${ }^{1}$, Franca Uche Richard $^{2}$, Mairama Abba Wulgo ${ }^{1}$

\author{
${ }^{1}$ Department of Crop Protection, Faculty of Agriculture, P. M. B.1069 University of Maiduguri, Borno State of Nigeria \\ ${ }^{2}$ Department of Animal Science, Faculty of Agriculture, P. M. B. 1069 Maiduguri, Nigeria
}

\author{
DOI: $10.36347 /$ sjavs.2022.v09i02.001 \\ | Received: 29.10.2021 | Accepted: 02.12.2021 | Published: 13.02.2022 \\ *Corresponding author: Richard Isa Bwala \\ Department of Crop Protection, Faculty of Agriculture, P. M. B.1069 University of Maiduguri, Borno State of Nigeria
}

Abstract

Field experiment was conducted in 2013 and 2014 cropping seasons at the Teaching and Research Farm of the Federal University of Agriculture Makurdi, Benue State of Nigeria to Assess the incidence of early and late leaf spot disease infection induced by (Cercospora arachicola Hori) and (Phaeoisariopsis persanata [Berk and Curt]) and agronomic performance of some selected groundnuts cultivars in Makurdi, Benue State of Nigeria. These selected groundnut cultivars (treatments) include: Ex-Dakar, Danbomboyo, Borno Brown, Samnut-10, Borno Red, Benue-AGR, Shar-nya, Aloshi, Samnut-22, Tisha-1, Ijiwanda, Sannut-21 and Campalla. The treatments were replicated four times and laid in a randomized complete block design (RCBD). The land and ridges were prepared manually using hoes. The seeds were sown by dibbling per hole on top of four ridges per a plot, each measuring $3 \mathrm{x}$ $2 \mathrm{~m}$. Results revealed significant difference $(\mathrm{P} \leq 0.05)$ on incidences of early and late leaf spot disease, plant height $(\mathrm{cm})$, canopy spread, pod and haulm yield among the groundnut cultivars evaluated in the two cropping seasons. The incidence of early leaf spot from 44 to 51 DAS and late leaf spot from 59 to 66 DAS were significantly different among the groundnut cultivars. In the first season, Samnut-10 and Samnut-21 did not show symptom of early leaf spot disease at 45 DAS and recorded the lowest incidence of early spot disease at $52 \mathrm{DAS}$; and also the least incidence of late leaf spot disease from 59 to 66 DAS compared to other cultivars respectively. In the second season, Campalla, Samnut-10 and Samnut-21 did not show symptom of early leaf spot disease at 45 DAS and had the lowest early leaf spot disease at 52 DAS. The lowest late leaf spot incidence was observed among Samnut-10 and Samnut-21 from 59 to 66 DAS. Tish-1 had the tallest plant height and widest canopy spread from 55 to 75 DAS in the first season and second season, whereas the widest canopy spread was observed among Campalla, Tisha-1, Aloshi, Sanut-10 and Samnut-21 at 75 DAS in the second season compared with the other cultivars In the first season, Samnut-10 and Samnut-21 had higher dry pods yield while in the second season Samnu-22 significantly had the highest dry pod yield compared with the others groundnut cultivars. Higher haulms (fodder) yield production trait was observed among Borno Brown, Ex-Daker, Campalla, Samnut-21, Danbonboyo, Samnut-22, Tisha-1 and Samnut-10. Hence they are recommended for introduction to other groundnut producing areas where groundnut haulms are highly valued as source of profitable income and fodder for farm animals. Lastly, Samnut-10, Samnut-21 and Samnut-22 exhibited lower incidences to the two leaf spot diseases and also higher pod yield characteristics across the seasons. Tisha-1, Campalla and Borno Brown were moderate in their reaction to the disease across the two cropping seasons and have exhibited a good prospect for genetic improvement for high yield production and for the management of groundnut diseases in this area.

Keywords: Groundnut cultivars, Early leaf spot, Late leaf spot, Disease incidence, yield.

Copyright $\odot 2022$ The Author(s): This is an open-access article distributed under the terms of the Creative Commons Attribution 4.0 International License (CC BY-NC 4.0) which permits unrestricted use, distribution, and reproduction in any medium for non-commercial use provided the original author and source are credited.

\section{INTRODUCTION}

Groundnut (Arachis hypogeae L.) is among the profitable economic cash crops grown by majority of lower income farmers in Benue State and other parts of Nigeria for revenue generation and its nutritional values. In Nigeria, the major groundnut producing states are Bauchi, Adamawa, Yobe and Borno in the Northeast; Taraba, Nasarawa, Plateau, Benue, Niger, Kwara, Kogi and FCT Abuja in the Central zone; and
Katsina, Jigawa, Kano, Sokoto, Zamfara, Kebbi and Kaduna in the Northwest [1]. The crop is an indeterminate and self-pollinated herbaceous legume which is usually used as rotational crop to maintain the soil nutrient level. Groundnut originated between Southern Bolivia and Northern Argentina in South America and was probably taken from Brazil to Africa, India and far East by the Portuguese explorers [2]. The crop is grown worldwide as food crop and for commercial purposes in several countries. Worldwide, 
about 23.95 million hectares of land were devoted to groundnut cultivation with the total production of 36.45 million tones and an average yields of $1520 \mathrm{~kg} / \mathrm{ha}$ [3]. Asia and Africa produced about $90 \%$ of the world's production of groundnut [4].

It can be successfully grown in the Tropical and Sub-Tropical winter rainfall environment and also in temperate humid climate with sufficient long warm summer. The crops require an average rainfall of 600$1200 \mathrm{~mm}$ per year but perform well where rainfall is between 500 and $700 \mathrm{~mm}$ per year is recorded in the Semi-arid region [5]. It crop performs better in soil $\mathrm{PH}$ of $6.0-6.5$ [6] with an optimum temperature of $25^{\circ} \mathrm{C}-$ $30^{\circ} \mathrm{C}$ [2]. Economically and nutritionally, groundnut is a very good source of income, the haulms are used as fodder for farm animals, the oil is used for cooking and preparation of various useful products such as margarines, soaps, butter, Pharmaceuticals, cosmetic, lubricant for motor engines, as fuel for diesel engines and emulsion for insecticides in the industries [6]. The seeds contain about oil $(48.32 \%)$, protein $(22-25 \%)$, carbohydrate $(20 \%)$, fiber $(5 \%)$ and ash and vitamins $\mathrm{B}$, $\mathrm{E}$ and $\mathrm{K}$ [7], it also contain some important mineral element such as magnesium, calcium and phosphorus [8].

The world largest producers of groundnut are China, India and USA [9]. Similarly [10], reported that the world production was 39.34 million tons with India as the world's leading producer between 2013 and 2014. Higher groundnut producers in Africa are Nigeria, Senegal, Sudan and Ghana respectively [11]. Nigeria groundnut production has been negligibly low compared with other developed countries and several biotic and abiotic factors responsible for dwindling in groundnut yield [12] reported low pod yield of groundnut in the developing countries which averages between 0.9 to $1.0 \mathrm{t} / \mathrm{ha}$ compared to the average of 1.6 t/ha of the global yield. Among the biotic constraints, early (Cercospora arachidocola Hori) and late leaf spot (Phaeoisariopsis personanta) are major destructive diseases of groundnut production due to defoliation of leaves and can cause $50 \%$ or more reduction in pod yield [13]. Similarly, [14] reported that shedding of infected leaves upon disease progression can lead to complete defoliation in susceptible genotypes and up to $70 \%$ yield loss. The symptoms of early leaf spots are brown to reddish brown in color and always have a yellow halo but with a raised surface, while the late leaf spots are dark brown to black spots without yellow halo but with rough and tufted appearance [15]. These fungal pathogens are soil-borne and capable of infecting almost every parts of the crop, thereby causing lesions on leaves, petioles, pegs main stem and lateral branches [16].

In the developed countries majority of the farmers' utilized synthetic fungicides very often for the management of groundnut diseases because of its effectiveness, but in the developing countries in contrary, the expensiveness of synthetic pesticides and shortage or lack of access to improved seed materials is presently a big production challenge. Besides the high cost of these conventional pesticides, their detrimental effects on human beings and the ecosystem are another major source of concern. This therefore necessitate continues search for safe control measures that can be easily adopted by the rural poor-resource farmers for the management of crop diseases with reduced cost and without harm to human beings and the environment. The aim of this research was to assess the incidence of Cercospora spp induced leaf spots disease infection on selected groundnuts cultivars in Makurdi, Benue State of Nigeria.

\section{MATERIAL AND METHODS}

Field experiment was conducted in two cropping seasons to assess the incidence of early and late leaf spot disease at the Teaching and Research Farm of the College of Agronomy, Federal University of Agriculture Makurdi Benue state located in the Southern Guinea Savanna of Nigeria. The experimental field has been under intensive cultivation of different leguminous and cereal crops for several which therefore ensures adequate source of inoculum in that disease hotspot. The thirteen treatments (groundnut cultivars) used for this experiment include: Samnut-22, Samnut-10 and Samnut-21 were obtained from the Institute for Agricultural Research (IAR) Zaria, Kaduna State; Borno Red, Danbomboyo, Borno Brown, Ex-Dakar and Campalla were obtained from the Department of Crop Protection, University of Maiduguri granary in Borno State; Aloshi and Tisha-1 were obtained from Nasarawa State and while Ijiwanda, Benue-AGR and Shar-nya were obtained from Benue State Agricultural and Rural Development Authority (BNARDA) all in Nigeria. The experimental field was prepared manually using local farming equipment. Cutlass was used for clearing the land while native hoes were used for preparing the ridges. The experimental area used measured $40 \mathrm{~m} \mathrm{x}$ $17 \mathrm{~m}=680.0 \mathrm{M}^{2}$. These thirteen cultivars were laid out in plots each measuring $3 \mathrm{~m} \times 2 \mathrm{~m}$ with four ridges and replicated four times in a randomized complete block design (RCBD). Two groundnut seeds were sown per hole with $20 \mathrm{~cm}$ and $75 \mathrm{~cm}$ intra and inter spacing between plant stands respectively. There were $1 \mathrm{~m}$ and $0.5 \mathrm{~m}$ as walking alleys between the replicates and rows respectively. The experimental area was kept weed-free throughout out the research period.

\section{Data Collection \\ Disease Assessment}

The appearance (incidence) of early leaf spot disease was assessed at 45 and 52 DAS, whereas that of the late leaf spot disease was assessed at 59 and 66 DAS in the middle rows of each plot. The percentage of disease incidence was assessed by counting the number of diseased plants in the two middle rows of each plot 
divided by the total number of plants (infected and uninfected plants) in the middle rows and then multiplied by one hundred using the disease incidence formula below:

Disease incidence $(\%)=$ Number of disease infected plants in the two middle rows $\times 100$

Total number of plants in the two middle rows (Healthy and diseased)

\section{Plant height and Canopy spread (cm)}

Ten plant stands were randomly selected and tagged in the two middle rows of each plot. The plant height and canopy spread were taken using a standard 1 -meter rule at 55, 65 and 75 DAS. The average of plant height $(\mathrm{cm})$ and canopy spread $(\mathrm{cm})$ of each net plot were respectively summed up and divided by ten.

\section{Dry pod and haulm weight (kg/ha)}

At physiological maturity stage of the crop, matured groundnut pods from the two middle rows of each plot were picked separately, cleaned, washed and sun-dry for two weeks and weighed separately using a sensitive electronic weighing scale model (Sartorius 6 $\mathrm{MBH}$ Gottingen) in the Plant Pathology Laboratory of Crop and Environmental Protection. Haulms from the two middle rows of each plot were weighed separately using a standard weighing balance scale. The values recorded per net-plot were converted to kilogram per hectare before statistical analysis.

\section{Data Analysis}

Data collected during the two cropping seasons were subjected to One-Way statistical Analyses of Variance (ANOVA) using SAS [17]. The mean separation was done using Duncan's New Multiple Range Test (DNMRT) at 5\% level of probability.

\section{RESULTS}

Incidence of Early Leaf Spot Disease on Selected Groundnut Cultivars at 45 and 52 DAS in the First and Second Cropping Seasons

The results on incidence of early leaf spot disease among the selected groundnut cultivars at 45 and 52 DAS in first and second cropping seasons are presented in Table 1. There was significant difference $(\mathrm{P} \leq 0.05)$ on early leaf spot disease incidences among the selected groundnut cultivars at 45 DAS and 52 DAS in the first and second seasons. Higher early leaf spot incidences were recorded among Ex-Dakar (28.02\%), Danbomboyo (25.39\%), Borno Brown (23.60\%) and Shar-nya $(20.76 \%)$, this was followed by Benue AGR $(19.72 \%)$ and Ijiwanda (19.11\% whereas Samnut-10 $(0.0 \%)$ and Samnut-21(\%) recorded the lowest disease incidence compared to others cultivars at 45 DAS. Similarly, higher disease incidences were observed among Benue AGR (48.28\%), Ex-Dakar (42.90\%), Danbomboyo (42.83\%) and Shar-nya 40.0) DAS, followed by Aloshi (38.55\%), Borno Red (38\%) and Ijiwanda (38. 38) whereas Samnut-10 (0.0\%) and Samnut-21 $(0.0 \%)$ had the lowest incidence of the disease symptom compared to other groundnut cultivars at 52 DAS.

In the second season, Ijiwanda $(41.89 \%)$ and Danbomboyo (39.69\%) significantly recorded higher early leaf spot disease incidence, followed by Benue AGR (31.27\% and Aloshi (25.63\%), while Campalla $(0.0 \%)$, Samnut-10 (0.0\%) and Samnut-21 (0.0\%) recorded the lowest disease incidences at 45 DAS respectively. At 52 DAS, higher disease incidences were recorded among Aloshi (51.25\%), Benue AGR (48.60\%) and Ijiwanda (48.48\%), this was followed by Shar-nya (47.39\%), Danbomboyo (46.28\% and ExDakar (44.39\%), while Samnut-10 (12.03\%), Samnut$21(13.44 \%)$ and Samnut-22 (15.41\%) recorded lower disease incidence compared to other cultivars.

Table 1: Incidence of Early Leaf Spot Disease at 45 and 52 DAS on Selected Groundnut Cultivars in the First and Second Cropping Seasons

\begin{tabular}{|c|c|c|c|c|}
\hline \multirow[t]{2}{*}{ Cultivars } & \multicolumn{2}{|c|}{ First Cropping Season } & \multicolumn{2}{|c|}{ Second Cropping Season } \\
\hline & 45 DAS & 52 DAS & 45 DAS & 52 DAS \\
\hline Aloshi & $12.92 \pm 2.88^{\mathrm{c}}$ & $38.55 \pm 1.58^{\mathrm{a}}$ & $25.63 \pm 4.80^{\mathrm{bc}}$ & $51.25 \pm 3.46^{\mathrm{a}}$ \\
\hline Benue-AGR & $19.72 \pm 4.86^{\mathrm{abc}}$ & $43.28 \pm 2.05^{\mathrm{a}}$ & $31.27 \pm 1.26^{\mathrm{b}}$ & $48.60 \pm 1.80^{\mathrm{a}}$ \\
\hline Borno Brown & $23.60 \pm 3.31^{\mathrm{ab}}$ & $38.19 \pm 3.94^{\mathrm{a}}$ & $23.44 \pm 2.91^{\mathrm{c}}$ & $35.34 \pm 1.40^{\mathrm{c}}$ \\
\hline Borno Red & $23.60 \pm 3.31^{\mathrm{ab}}$ & $38.19 \pm 3.94^{\mathrm{a}}$ & $13.41 \pm 1.00^{\mathrm{d}}$ & $41.35 \pm 1.38^{\mathrm{bc}}$ \\
\hline Campalla & $10.28 \pm 0.28^{\mathrm{c}}$ & $13.61 \pm 1.44^{b}$ & $0.00 \pm 0.00^{\mathrm{e}}$ & $18.22 \pm 1.18^{\mathrm{e}}$ \\
\hline Danbomboyo & $25.39 \pm 2.42^{\mathrm{a}}$ & $42.83 \pm 2.90^{\mathrm{a}}$ & $39.69 \pm 1.71^{\mathrm{a}}$ & $46.28 \pm 2.26^{\mathrm{ab}}$ \\
\hline Ex-Dakar & $28.02 \pm 3.58^{\mathrm{a}}$ & $42.90 \pm 2.14^{\mathrm{a}}$ & $18.30 \pm 4.67^{\mathrm{cd}}$ & $44.39 \pm 1.64^{\mathrm{ab}}$ \\
\hline Ijiwanda & $19.11 \pm 3.36^{\mathrm{abc}}$ & $38.38 \pm 2.51^{\mathrm{a}}$ & $41.89 \pm 3.39^{\mathrm{a}}$ & $48.48 \pm 1.68^{\mathrm{a}}$ \\
\hline Samnut-10 & $0.00 \pm 0.00^{\mathrm{d}}$ & $0.00 \pm 0.00^{c}$ & $0.00 \pm 0.00^{\mathrm{e}}$ & $12.03 \pm 0.98^{\mathrm{e}}$ \\
\hline Samnut-21 & $0.00 \pm 0.00^{\mathrm{d}}$ & $0.00 \pm 0.00^{c}$ & $0.00 \pm 0.00^{\mathrm{e}}$ & $13.44 \pm 2.22^{\mathrm{e}}$ \\
\hline Samnut-22 & $11.75 \pm 1.18^{\mathrm{c}}$ & $14.65 \pm 4.29^{b}$ & $11.64 \pm 1.56^{\mathrm{d}}$ & $15.41 \pm 1.67^{\mathrm{e}}$ \\
\hline Shar-nya & $20.76 \pm 3.74^{\mathrm{abc}}$ & $40.00 \pm 3.24^{\mathrm{a}}$ & $21.14 \pm 2.33^{c}$ & $47.39 \pm 2.69^{\mathrm{ab}}$ \\
\hline Tisha-1 & $14.72 \pm 5.34^{\mathrm{bc}}$ & $16.03 \pm 2.46^{b}$ & $11.35 \pm 0.09^{\mathrm{d}}$ & $26.00 \pm 3.92^{\mathrm{d}}$ \\
\hline$P$-Value & $\leq 0.05$ & $\leq 0.05$ & $\leq 0.05$ & $\leq 0.05$ \\
\hline$C V(\%)$ & 15.34 & 25.41 & 17.12 & 8.69 \\
\hline
\end{tabular}

Mean values within each column with the same letter (s) are not significantly different $(\mathrm{P} \leq 0.05)$ from each other according to Duncan's New Multiple Rang Test (DNMRT). CV = Coefficient of variation, DAS $=$ Das after sowing 
Incidence of Late Leaf Spot Disease on Selected Groundnut Cultivars at 59 and 66 DAS in the First and Second Cropping Seasons

The results on incidence of late leaf spot disease among the selected groundnut cultivars at 59 and 66 DAS in the First and second cropping seasons are presented in Table 2. There was significant difference $(\mathrm{P} \leq 0.05)$ on incidences of late leaf spot disease among the selected groundnut cultivars at 59 DAS and 66 DAS in both seasons. At 59 DAS of the first season, higher late leaf spot disease incidence which were not significantly different from each other were recorded among Ex-Dakar (56.53\%), Benue AGR $(56.10 \%)$, Shar-nya $(55.31 \%)$, Ijiwanda $(53.15 \%)$ and Aloshi $(51.51 \%)$, while the lowest disease incidence were recorded among Samnut-10 (16.35\%) and Samnut-21 (16.18\%) respectively. Similarly, at 66 DAS, higher disease incidences were recorded among Ijiwanda $(93.61 \%)$ and Shar-nya $(92.30 \%)$, this was followed by Ex-Dakar (87.48\%), Aloshi (80.77\%), Benue AGR (78.38\%) and Borno Red (77.98\%) but which did not statistically vary from each other. Lower disease incidence was observed on Samnut-21 (31.59\%), followed by Samnut-22 (44.87\%) and Samnut-10 (47.72\%) compare to other groundnut cultivars at 66 DAS respectively.

In the second season, results at 59 DAS showed that Shar-nya $(87.38 \%)$ recorded higher late leaf spot disease incidence but it was not significantly different from disease incidences of $79-84.01 \%$ recorded among Danbomboyo, Benue AGR, Ijiwanda and Ex-Dakar. The lowest incidence of the disease was observed on Samnut-10 (16.53\%), followed by Samnut$21(20.31 \%)$ and Samnut-22 (37.18\%) at 59 DAS accordingly. On the same trend, at 66 DAS, higher disease incidence which range from $90-96.75 \%$ was recorded among Benue AGR, Borno Red, Aloshi, Danbomboyo, Shar-nya, Ijiwanda and Ex-Dakar while Tisha-1, Samnut-22 and Borno Brown recorded moderately disease incidence which range from 55 $57.75 \%$. Lower incidence of the disease was observed among Samnut-21 (35.82\%) and Samnut-10 (42.95\%) compare to other cultivars at 66 DAS respectively.

Table 2: Incidence of Late Leaf Spot Disease at 59 and 66 DAS on Selected Groundnut Cultivars in the First and Second Cropping Seasons

\begin{tabular}{|l|l|l|l|l|}
\hline Cultivars & First Cropping Season & \multicolumn{3}{l|}{ Second Cropping Season } \\
\hline & $\mathbf{5 9}$ DAS & 66 DAS & 59 DAS & 66 DAS \\
\hline Aloshi & $51.51 \pm 2.98^{\mathrm{a}}$ & $80.77 \pm 6.73^{\mathrm{abc}}$ & $68.56 \pm 5.10^{\mathrm{c}}$ & $92.00 \pm 4.54^{\mathrm{a}}$ \\
\hline Benue-AGR & $56.10 \pm 1.24^{\mathrm{a}}$ & $78.38 \pm 8.08^{\mathrm{abc}}$ & $82.64 \pm 2.80^{\mathrm{ab}}$ & $90.41 \pm 2.78^{\mathrm{a}}$ \\
\hline Borno Brown & $51.41 \pm 1.47^{\mathrm{b}}$ & $77.98 \pm 4.22^{\mathrm{abc}}$ & $45.04 \pm 2.15^{\mathrm{de}}$ & $57.75 \pm 2.95^{\mathrm{b}}$ \\
\hline Borno Red & $51.41 \pm 1.47^{\mathrm{b}}$ & $77.98 \pm 4.22^{\mathrm{abc}}$ & $77.19 \pm 2.99^{\mathrm{b}}$ & $91.25 \pm 3.14^{\mathrm{a}}$ \\
\hline Campalla & $43.05 \pm 2.66^{\mathrm{bc}}$ & $58.28 \pm 3.84^{\mathrm{d}}$ & $43.21 \pm 1.72^{\mathrm{de}}$ & $54.75 \pm 2.62^{\mathrm{b}}$ \\
\hline Danbomboyo & $50.88 \pm 1.43^{\mathrm{b}}$ & $76.50 \pm 4.05^{\mathrm{bc}}$ & $79.32 \pm 2.31^{\mathrm{ab}}$ & $92.75 \pm 4.38^{\mathrm{a}}$ \\
\hline Ex-Dakar & $56.53 \pm 2.65^{\mathrm{a}}$ & $87.48 \pm 4.45^{\mathrm{abc}}$ & $84.01 \pm 2.55^{\mathrm{ab}}$ & $96.75 \pm 1.97^{\mathrm{a}}$ \\
\hline Ijiwanda & $53.15 \pm 1.44^{\mathrm{a}}$ & $93.61 \pm 3.79^{\mathrm{a}}$ & $83.43 \pm 1.74^{\mathrm{ab}}$ & $95.79 \pm 1.94^{\mathrm{a}}$ \\
\hline Samnut-10 & $16.35 \pm 1.96^{\mathrm{e}}$ & $47.72 \pm 1.15^{\mathrm{d}}$ & $16.53 \pm 2.96^{\mathrm{f}}$ & $42.95 \pm 1.15^{\mathrm{c}}$ \\
\hline Samnut-21 & $16.18 \pm 1.81^{\mathrm{e}}$ & $31.59 \pm 3.86^{\mathrm{e}}$ & $20.31 \pm 1.29^{\mathrm{f}}$ & $35.82 \pm 1.77^{\mathrm{c}}$ \\
\hline Samnut-22 & $37.68 \pm 1.49^{\mathrm{cd}}$ & $44.87 \pm 1.49^{\mathrm{de}}$ & $37.18 \pm 1.25^{\mathrm{e}}$ & $57.00 \pm 2.94^{\mathrm{b}}$ \\
\hline Shar-nya & $55.31 \pm 2.10^{\mathrm{a}}$ & $92.30 \pm 3.24^{\mathrm{a}}$ & $87.38 \pm 2.33^{\mathrm{a}}$ & $95.30 \pm 2.72^{\mathrm{a}}$ \\
\hline Tisha-1 & $36.44 \pm 2.52^{\mathrm{d}}$ & $75.41 \pm 5.60^{\mathrm{c}}$ & $48.47 \pm 5.08^{\mathrm{d}}$ & $55.82 \pm 2.30^{\mathrm{b}}$ \\
\hline$P$-Value & $\leq 0.05$ & $\leq 0.05$ & $\leq 0.05$ & $\leq 0.05$ \\
\hline CV(\%) & 19. & 23.10 & 11.23 & 20.13 \\
\hline
\end{tabular}

Mean values within each column with the same letter $(\mathrm{s})$ are not significantly different $(\mathrm{P} \leq 0.05)$ from each other according to Duncan's New Multiple Rang Test (DNMRT). CV = Coefficient of variation, DAS= Das after sowing

Plant Height (cm) of the Selected Groundnut Cultivars at 55, 65 and 75 DAS in the First and Second Cropping Seasons

Results on plant height of selected groundnut cultivars at 55, 65 and 75 DAS in the first and second cropping seasons are presented in Table 3 . The results of the two cropping seasons indicated that there were significant differences $(\mathrm{P} \leq 0.05)$ on plant height among the selected groundnut cultivars from 55 to 75 DAS.

In the first cropping season, result at 55 DAS showed that Tisha-1 $(20.30 \mathrm{~cm})$ recorded the highest plant height followed by Aloshi $(19.33 \mathrm{~cm})$ and Samnut-
22 (19.05) but which did no significantly vary from each other. Samnut-10 (14.20cm) and Samnut$21(14.72 \mathrm{~cm})$ recorded lower plant height compared to other cultivars respectively. Similarly, at 65 DAS Tisha-1 $(27.44 \mathrm{~cm})$ significantly recorded higher plant height followed also by Alosh $(26.08 \%)$, Campalla $(25.29 \%)$ and Samnut-22 $(25.31 \%)$, while the lowest plant height was observed among Samnut-10 $(18.02 \mathrm{~cm})$, Borno Red $(18.41 \mathrm{~cm})$, and Samnut-21 $(18.68 \mathrm{~cm})$ compared to other groundnut cultivars. Also, higher plant height was recorded on Tisha-1 $(40.28 \mathrm{~cm})$, followed by Campalla $(38.82 \mathrm{~cm})$ and Aloshi $(38.53)$ which did not statistically vary from each other 
respectively at 75 DAS. Statistically, lower plant height was observed among Samnut-10 $(23.16 \mathrm{~cm})$ and Samnut-21 $(23.65 \mathrm{~cm})$ at 75 DAS respectively.

In the second cropping season, results at 55 DAS showed that Tisha-1 $(29.74 \mathrm{~cm})$ recorded higher plant height followed by Aloshi $(28.55 \mathrm{~cm})$ and Benue AGR $(27.36 \mathrm{~cm})$ while Samnut-10 $(16.0 \mathrm{~cm})$ and Samnut-21 $(18.18 \mathrm{~cm})$ had the lower compared to other the cultivars. At 65 DAS, higher plant height was observed among Tisha-1 $(33.56 \mathrm{~cm})$, Aloshi $(31.33 \mathrm{~cm})$,
Benue-AGR (30. 64cm), Campalla $(30.63 \mathrm{~cm})$, ExDaker $(30.15 \mathrm{~cm})$. lower plant height was observed among Samnut-10 $(18.69 \mathrm{~cm})$ and Samnut-21 $(22.03 \mathrm{~cm})$ compared to other cultivars at 65 DAS respectively. Similarly, at 75 DAS, Tisha-1 $(44.65 \mathrm{~cm})$ recorded higher plant height which did not vary significantly from those observed from Aloshi $(42.57 \mathrm{~cm})$, Campalla $(42.61 \mathrm{~cm})$ and Benue-AGR $(40.95 \mathrm{~cm})$ respectively. Samnut-10 (25.61 cm) and Samnut-21 (28.12cm) recorded lower plant height compared to other groundnut cultivars at 75 DAS respectively.

Table 3: Plant Height (cm) of the Selected Groundnut Cultivars at 55, 65 and 75DAS in the First and Second Cropping Seasons

\begin{tabular}{|c|c|c|c|c|c|c|}
\hline Cultivars & \multicolumn{3}{|c|}{ First Cropping Season } & \multicolumn{3}{|c|}{ Second Cropping Season } \\
\hline & 55 DAS & 65 DAS & 75 DAS & 55 DAS & 65 DAS & 75 DAS \\
\hline Aloshi & $19.33 \pm 0.57^{\mathrm{ab}}$ & $26.08 \pm 2.15^{\mathrm{ab}}$ & $38.53 \pm 0.74^{\mathrm{ab}}$ & $28.55 \pm 1.89^{\mathrm{ab}}$ & $31.33 \pm 1.66^{\mathrm{ab}}$ & $42.57 \pm 2.22^{\mathrm{a}}$ \\
\hline Benue-AGR & $15.81 \pm 1.05^{\mathrm{bcd}}$ & $21.26 \pm 1.99^{\mathrm{abc}}$ & $30.35 \pm 3.84^{\mathrm{bcd}}$ & $27.36 \pm 0.52^{\mathrm{ab}}$ & $30.64 \pm 1.59^{\mathrm{abc}}$ & $40.95 \pm 0.95^{\mathrm{a}}$ \\
\hline Borno Brown & $17.58 \pm 0.72^{\mathrm{abcd}}$ & $21.86 \pm 0.93^{\mathrm{abc}}$ & $27.97 \pm 0.83^{\mathrm{de}}$ & $19.32 \pm 0.99^{c}$ & $22.71 \pm 1.39^{\mathrm{de}}$ & $33.28 \pm 0.76^{\text {bcd }}$ \\
\hline Borno Red & $15.42 \pm 1.17^{\mathrm{cd}}$ & $18.41 \pm 1.72^{\mathrm{c}}$ & $28.49 \pm 2.31^{\mathrm{de}}$ & $24.25 \pm 1.09^{b}$ & $27.54 \pm 0.62^{\mathrm{bc}}$ & $31.58 \pm 0.51^{\text {cde }}$ \\
\hline Campalla & $17.61 \pm 2.18^{\mathrm{abcd}}$ & $25.61 \pm 3.24^{\mathrm{ab}}$ & $38.82 \pm 2.77^{\mathrm{abc}}$ & $25.18 \pm 1.31^{\mathrm{ab}}$ & $30.63 \pm 0.84^{\mathrm{abc}}$ & $42.61 \pm 1.37^{\mathrm{a}}$ \\
\hline Danbomboyo & $17.21 \pm 0.61^{\mathrm{abcd}}$ & $21.29 \pm 0.84^{\mathrm{abc}}$ & $28.30 \pm 0.68^{\mathrm{de}}$ & $25.19 \pm 0.85^{\mathrm{ab}}$ & $28.25 \pm 0.71^{\mathrm{bc}}$ & $35.34 \pm 0.86^{\mathrm{bc}}$ \\
\hline Ex-Daker & $17.12 \pm 0.74^{\mathrm{abcd}}$ & $22.72 \pm 1.31^{\mathrm{abc}}$ & $29.37 \pm 1.01^{\mathrm{de}}$ & $26.31 \pm 1.76^{\mathrm{ab}}$ & $30.15 \pm 1.09^{\mathrm{abc}}$ & $36.00 \pm 1.01^{\mathrm{bc}}$ \\
\hline Ijiwanda & $16.13 \pm 1.65^{\text {bcd }}$ & $19.99 \pm 2.82^{\mathrm{bc}}$ & $28.61 \pm 2.41^{\mathrm{de}}$ & $26.82 \pm 1.37^{\mathrm{ab}}$ & $29.41 \pm 1.34^{\mathrm{abc}}$ & $36.62 \pm 0.29^{b}$ \\
\hline Samnut-10 & $14.20 \pm 0.42^{\mathrm{d}}$ & $18.02 \pm 0.71^{\mathrm{c}}$ & $23.16 \pm 0.49^{\mathrm{e}}$ & $16.00 \pm 0.33^{\mathrm{c}}$ & $18.69 \pm 0.52^{\mathrm{e}}$ & $25.61 \pm 0.93^{f}$ \\
\hline Samnut-21 & $14.72 \pm 1.18^{d}$ & $18.68 \pm 2.09^{c}$ & $23.65 \pm 0.96^{\mathrm{e}}$ & $18.18 \pm 1.23^{\mathrm{c}}$ & $22.03 \pm 1.07^{\mathrm{e}}$ & $28.12 \pm 0.94^{\mathrm{ef}}$ \\
\hline Samnut-22 & $19.05 \pm 0.98^{\mathrm{abc}}$ & $25.31 \pm 1.69^{\mathrm{ab}}$ & $33.15 \pm 1.29^{\mathrm{cd}}$ & $24.76 \pm 0.60^{b}$ & $26.83 \pm 0.86^{\mathrm{bcd}}$ & $30.66 \pm 0.61^{\text {de }}$ \\
\hline Shar-nya & $17.02 \pm 0.81^{\mathrm{abcd}}$ & $22.52 \pm 1.68^{\mathrm{abc}}$ & $27.82 \pm 2.18^{\mathrm{de}}$ & $24.06 \pm 2.00^{\mathrm{b}}$ & $26.46 \pm 2.40^{\mathrm{cd}}$ & $32.34 \pm 2.78^{\text {bcde }}$ \\
\hline Tisha-1 & $20.30 \pm 1.48^{\mathrm{a}}$ & $27.44 \pm 2.76^{\mathrm{a}}$ & $40.28 \pm 2.60^{\mathrm{a}}$ & $29.74 \pm 2.57^{\mathrm{a}}$ & $33.56 \pm 2.63^{\mathrm{a}}$ & $44.65 \pm 2.78^{\mathrm{a}}$ \\
\hline P-Value & $<0.01$ & 0.01 & $<0.01$ & 0.04 & $<0.01$ & $<0.01$ \\
\hline$C V$ & 18.97 & 26.41 & 20.17 & 21.32 & 20.16 & 20.31 \\
\hline
\end{tabular}

Mean values within each column with the same letter $(\mathrm{s})$ are not significantly different $(\mathrm{P} \leq 0.05)$ from each other according to Duncan's New Multiple Rang Test (DNMRT). CV = Coefficient of variation, DAS= Das after sowing

\section{Canopy spread among the selected Groundnut Cultivars at 55, 65 and 75 DAS in First and Second Cropping Seasons}

Results on canopy spread among the selected groundnut cultivars at 55, 65 and 75 DAS in the first and second cropping seasons are presented in Table 4. The results of both seasons showed that there were significant differences $(\mathrm{P} \leq 0.05)$ on canopy spread among the selected groundnut cultivars spread from 55 to 75 DAS.

In the first season 2011, result at 55 DAS showed that the widest canopy spread was recorded on Tisha-1 $(29.54 \mathrm{~cm})$ followed by Campalla $(27.39 \mathrm{~cm})$ Samnut-22 $(27.34 \mathrm{~cm})$ and Danbomboyo $(27.06 \mathrm{~cm})$, while the least canopy spread was observed among Borno Red $(21.82 \mathrm{~cm})$ and Shar-nya $(23.75 \mathrm{~cm})$ compare to other cultivars respectively. At 65 DAS, wider canopy spread recorded among Tisha-1 $(49.80 \mathrm{~cm})$ and Campalla $(47.39 \mathrm{~cm})$ but which did not statistically vary from each other, while the least canopy spread was recorded on Borno Red $(30.24 \mathrm{~cm})$ followed by ExDaker $(36.35 \mathrm{~cm})$, Shar-nya $(36.96 \mathrm{~cm})$ and Samnut-10 $(37.47 \mathrm{~cm})$ compare to other groundnut cultivars accordingly. Similarly at 75 DAS, wider canopy spread were observed among Tisha-1 $(53.44 \mathrm{~cm})$, Campalla $(52.98 \mathrm{~cm})$ and Samnut-22 $(51.05 \mathrm{~cm})$, while the least was recorded on Borno Red $(37.20 \mathrm{~cm})$ followed by Shar-nya $(40.78 \mathrm{~cm})$ and Ijiwanda $(42.17 \mathrm{~cm})$ compare to other cultivars respectively.

In the second season, results at revealed that at 55 DAS, Tisha-1 $(44.23 \mathrm{~cm})$ recorded the widest canopy spread followed by Samnut-22 $(41.48 \mathrm{~cm})$, while Borno Brown $(36.58 \mathrm{~cm})$, Ex-Daker $(35.49 \mathrm{~cm})$ and Ijiwanda $(36.69 \mathrm{~cm})$ recorded lesser and statistically similar canopy spread compare to other cultivars respectively. At 65 DAS, Tisha-1 $(50.17 \mathrm{~cm})$ and Campalla $(50.74 \mathrm{~cm})$, followed by Aloshi $(49.19 \mathrm{~cm})$ recorded the widest but statistically similar canopy spread, while ExDaker $(40.85 \mathrm{~cm})$, Borno brown $(41.91 \mathrm{~cm})$, Ijiwanda $(42.25 \mathrm{~cm})$ and Shar-nya $(42.15 \mathrm{~cm})$ had the least canopy spread. Similarly, at 75 DAS the widest canopy spread was observed among Campalla $(55.92 \mathrm{~cm})$, Samnut-10 $(54.88 \mathrm{~cm})$, Samnut-22 $(52.25 \mathrm{~cm})$, Aloshi $(54.13 \mathrm{~cm})$, and Tisha-1 $(54.01 \mathrm{~cm})$ whereas lesser canopy spread where recorded among Ex-Daker $(46.52 \mathrm{~cm})$, Ijiwanda $(46.52 \mathrm{~cm})$, Borno Brown $(46.76 \mathrm{~cm})$ and Borno Red $(46.71 \mathrm{~cm})$ compare toother groundnut cultivars respectively. 
Table 4: Canopy spread among the Selected Groundnut Cultivars at 55, 65 and 75 DAS in the First and Second Cropping Seasons

\begin{tabular}{|c|c|c|c|c|c|c|}
\hline \multirow[t]{2}{*}{ Cultivars } & \multicolumn{3}{|c|}{ First Cropping Season } & \multicolumn{3}{|c|}{ Second Cropping Season } \\
\hline & 55 DAS & 65 DAS & 75 DAS & 55 DAS & 65 DAS & 75 DAS \\
\hline Aloshi & $25.36 \pm 1.36^{\mathrm{abc}}$ & $43.69 \pm 4.38^{\mathrm{abc}}$ & $45.33 \pm 4.65^{\mathrm{abcd}}$ & $38.32 \pm 1.51^{\mathrm{ab}}$ & $49.19 \pm 1.15^{\mathrm{ab}}$ & $54.13 \pm 1.42^{\mathrm{ab}}$ \\
\hline Benue-AGR & $24.86 \pm 0.96^{\mathrm{bc}}$ & $40.08 \pm 2.62^{\mathrm{bc}}$ & $44.25 \pm 2.32^{\mathrm{bcd}}$ & $39.87 \pm 1.29^{\mathrm{ab}}$ & $44.23 \pm 0.92^{\mathrm{bc}}$ & $48.89 \pm 0.66^{\text {cde }}$ \\
\hline Borno Brown & $25.39 \pm 1.26^{\mathrm{abc}}$ & $37.85 \pm 2.86^{\mathrm{bcd}}$ & $42.65 \pm 2.80^{\mathrm{bcd}}$ & $36.58 \pm 1.74^{\mathrm{b}}$ & $41.91 \pm 1.84^{\mathrm{c}}$ & $46.76 \pm 1.38^{\mathrm{de}}$ \\
\hline Borno Red & $21.82 \pm 0.95^{\mathrm{c}}$ & $30.24 \pm 1.69^{\mathrm{d}}$ & $37.20 \pm 1.37^{\mathrm{d}}$ & $38.17 \pm 2.84^{\mathrm{ab}}$ & $43.72 \pm 1.46^{\mathrm{bc}}$ & $46.71 \pm 1.06^{\mathrm{de}}$ \\
\hline Campalla & $27.39 \pm 2.27^{\mathrm{ab}}$ & $47.39 \pm 4.12^{\mathrm{ab}}$ & $52.98 \pm 3.05^{\mathrm{a}}$ & $37.14 \pm 2.56^{\mathrm{ab}}$ & $50.74 \pm 1.36^{\mathrm{a}}$ & $55.92 \pm 0.61^{\mathrm{a}}$ \\
\hline Danbomboyo & $27.06 \pm 1.44^{\mathrm{ab}}$ & $40.87 \pm 2.56^{\mathrm{abc}}$ & $46.06 \pm 2.07^{\mathrm{abc}}$ & $37.85 \pm 0.48^{\mathrm{ab}}$ & $43.21 \pm 1.22^{\mathrm{c}}$ & $47.24 \pm 0.44^{\mathrm{de}}$ \\
\hline Ex-Daker & $24.55 \pm 0.44^{\mathrm{bc}}$ & $36.35 \pm 2.41^{\mathrm{cd}}$ & $44.53 \pm 2.12^{\mathrm{bcd}}$ & $35.49 \pm 3.17^{b}$ & $40.85 \pm 2.46^{\mathrm{c}}$ & $46.52 \pm 1.10^{\mathrm{e}}$ \\
\hline Ijiwanda & $24.69 \pm 1.25^{\mathrm{bc}}$ & $38.08 \pm 1.73^{\mathrm{bcd}}$ & $42.17 \pm 1.91^{\mathrm{cd}}$ & $36.69 \pm 2.16^{\mathrm{b}}$ & $42.25 \pm 1.53^{\mathrm{c}}$ & $46.52 \pm 1.10^{\mathrm{e}}$ \\
\hline Samnut-10 & $24.22 \pm 0.57^{\mathrm{bc}}$ & $37.47 \pm 0.67^{\mathrm{cd}}$ & $46.52 \pm 1.48^{\mathrm{abc}}$ & $38.58 \pm 1.52^{\mathrm{ab}}$ & $46.37 \pm 1.46^{\mathrm{abc}}$ & $54.88 \pm 1.83^{\mathrm{ab}}$ \\
\hline Samnut-21 & $25.37 \pm 1.77^{a b c}$ & $40.21 \pm 1.23^{b c}$ & $48.26 \pm 0.66^{\mathrm{abc}}$ & $36.17 \pm 2.40^{\mathrm{ab}}$ & $46.79 \pm 2.19^{\mathrm{abc}}$ & $50.71 \pm 1.70^{\mathrm{bcd}}$ \\
\hline Samnut-22 & $27.34 \pm 0.84^{\mathrm{ab}}$ & $43.64 \pm 3.78^{\mathrm{abc}}$ & $51.05 \pm 2.44^{\mathrm{ab}}$ & $41.48 \pm 2.80^{\mathrm{ab}}$ & $49.38 \pm 2.52^{\mathrm{ab}}$ & $52.25 \pm 2.18^{\mathrm{abc}}$ \\
\hline Shar-nya & $23.75 \pm 1.64^{\mathrm{bc}}$ & $36.96 \pm 1.90^{\mathrm{cd}}$ & $40.78 \pm 1.55^{\mathrm{cd}}$ & $37.28 \pm 2.53^{\mathrm{ab}}$ & $42.15 \pm 2.83^{c}$ & $47.24 \pm 2.33^{\mathrm{de}}$ \\
\hline Tisha-1 & $29.54 \pm 0.77^{\mathrm{a}}$ & $49.80 \pm 5.03^{\mathrm{a}}$ & $53.44 \pm 4.13^{\mathrm{a}}$ & $44.23 \pm 2.32^{\mathrm{a}}$ & $50.17 \pm 1.63^{\mathrm{a}}$ & $54.01 \pm 1.33^{\mathrm{ab}}$ \\
\hline$P$-Value & 0.02 & $<0.01$ & $<0.01$ & 0.04 & $<0.01$ & $<0.01$ \\
\hline$C V$ & 21.10 & 19.56 & 17.25 & 21.23 & 25.41 & 20.11 \\
\hline
\end{tabular}

Mean values within each column with the same letter $(\mathrm{s})$ are not significantly different $(\mathrm{P} \leq 0.05)$ from each other according to Duncan's New Multiple Rang Test (DNMRT). CV = Coefficient of variation, DAS= Das after sowing

\section{Dry Pod and Haulm Weight (kg/ha) of the Selected Groundnut Cultivars in The First and Second Cropping Seasons}

The results on dry pods and haulms weight $(\mathrm{kg} / \mathrm{ha})$ in the first and second cropping seasons are presented in Table 5. Results of the two cropping seasons revealed that there were significant difference $(\mathrm{P} \leq 0.05)$ among the evaluated groundnut cultivars on dry pods and haulm weight $(\mathrm{kg} / \mathrm{ha})$ respectively

In the first cropping season, the highest pod yield weight in $\mathrm{kg} / \mathrm{ha}$ was recorded among Samnut-10 $(1747.0 \mathrm{~kg})$ and Samnut-21 (1563.0kg) which did not statistically vary from each other, followed by Campalla (1426.0kg). Lower dry pod weight was observed among Benue AGR $(821.20 \mathrm{~kg})$, Borno Brown $(774.90 \mathrm{~kg})$ and Ijiwanda $(809.30 \mathrm{~kg})$ compared to other groundnut cultivars respectively. The highest haulm weight in $\mathrm{kg} / \mathrm{ha}$ which did not significantly differ from each other was recorded among Samnut-21 (5092.0kg), Samnut-10 $(4533.0 \mathrm{~kg})$ and Samnut-22 (4425.0kg), while the least haulm weight was recorded among Borno Red $(2325.0 \mathrm{~kg})$ and Shar-nya $(2367.0 \mathrm{~kg})$ compared to other cultivars.

In the second cropping season, Samnut-22 $(3415.0 \mathrm{~kg})$ significantly recorded the highest dry pod yield weight in $\mathrm{kg} / \mathrm{ha}$, this was followed by Tisha-1 $(2951.0 \mathrm{~kg})$, while the lowest dry pod yield weight in $\mathrm{kg} / \mathrm{ha}$ was recorded among Aloshi (799.50kg), Shar-nya (889.0kg), Danbomboyo (962.0kg), and Borno Brown $(1000.0 \mathrm{~kg})$ compared to other cultivars respectively. Significantly higher haulms weight in $\mathrm{kg} / \mathrm{ha}$ was recorded among Samnut-21 (4392.0kg), Samnut-10 $(4300.0 \mathrm{~kg})$, Samnut-22 (4192.0kg) and Borno Brown $(4192.0 \mathrm{~kg})$ but which did not statistically differ from each other accordingly, this was followed by Campalla $(4042.0 \mathrm{~kg})$. Lowest haulms weight in $\mathrm{kg} / \mathrm{ha}$ were recorded among Ex-Dakar $(2858.0 \mathrm{~kg})$ and Shar-nya $(2833.0 \mathrm{~kg})$ compared to other groundnut cultivars respectively

Table 5: Dry Pods and Haulms Weight (kg/ha) of the Selected Groundnut Cultivars in The First and Second Cropping Seasons

\begin{tabular}{|l|l|l|l|l|}
\hline Cultivars & \multicolumn{2}{|l|}{ First Cropping Season } & \multicolumn{2}{l|}{ Second Cropping Season } \\
\hline & $\begin{array}{l}\text { Dry pod weight } \\
(\mathbf{k g})\end{array}$ & $\begin{array}{l}\text { Haulm weight } \\
(\mathbf{k g})\end{array}$ & $\begin{array}{l}\text { Dry pod weight } \\
(\mathbf{k g})\end{array}$ & $\begin{array}{l}\text { Haulm weight } \\
(\mathbf{k g})\end{array}$ \\
\hline Aloshi & $1229.00 \pm 12.50^{\mathrm{c}}$ & $2967.00 \pm 201.00^{\mathrm{bcd}}$ & $799.50 \pm 20.80^{\mathrm{h}}$ & $3333.00 \pm 196.00^{\mathrm{abc}}$ \\
\hline Benue-AGR & $821.20 \pm 70.90^{\mathrm{e}}$ & $2975.00 \pm 320.00^{\mathrm{bcd}}$ & $1516.30 \pm 87.90^{\mathrm{e}}$ & $3033.00 \pm 377.00^{\mathrm{bc}}$ \\
\hline Borno Brown & $774.90 \pm 18.90^{\mathrm{e}}$ & $5267.00 \pm 424.00^{\mathrm{a}}$ & $1000.40 \pm 70.80^{\mathrm{fg}}$ & $4192.00 \pm 342.00^{\mathrm{a}}$ \\
\hline Borno Red & $863.80 \pm 35.50^{\mathrm{de}}$ & $2325.00 \pm 624.00^{\mathrm{d}}$ & $1344.00 \pm 11.20^{\mathrm{ef}}$ & $3325.00 \pm 659.00^{\mathrm{abc}}$ \\
\hline Campalla & $1426.60 \pm 58.40^{\mathrm{bc}}$ & $3817.00 \pm 818.00^{\mathrm{abcd}}$ & $1517.40 \pm 59.80^{\mathrm{e}}$ & $4042.00 \pm 283.00^{\mathrm{ab}}$ \\
\hline Danbomboyo & $1313.00 \pm 14.00^{\mathrm{bc}}$ & $4025.00 \pm 403.00^{\text {abcd }}$ & $962.00 \pm 12.30^{\mathrm{gh}}$ & $3358.00 \pm 130.00^{\mathrm{abc}}$ \\
\hline Ex-Dakar & $1136.70 \pm 85.30^{\mathrm{cd}}$ & $2767.00 \pm 137.00^{\mathrm{abcd}}$ & $1237.00 \pm 12.30^{\text {efg }}$ & $2858.00 \pm 362.00^{\mathrm{c}}$ \\
\hline Ijiwanda & $809.30 \pm 82.30^{\mathrm{e}}$ & $2800.00 \pm 123.00^{\text {bcd }}$ & $1501.00 \pm 12.50^{\mathrm{d}}$ & $3792.00 \pm 441.00^{\mathrm{abc}}$ \\
\hline
\end{tabular}


Richard Isa Bwala et al., Sch J Agric Vet Sci, Feb, 2022; 9(2): 14-22

\begin{tabular}{|l|l|l|l|l|}
\hline Samnut-10 & $1747.00 \pm 16.80^{\mathrm{a}}$ & $4533.00 \pm 766.00^{\mathrm{ab}}$ & $2133.00 \pm 17.80^{\mathrm{c}}$ & $4300.00 \pm 332.00^{\mathrm{a}}$ \\
\hline Samnut-21 & $1563.00 \pm 16.80^{\mathrm{ab}}$ & $5092.00 \pm 625.00^{\mathrm{a}}$ & $2509.50 \pm 76.20^{\mathrm{c}}$ & $4392.00 \pm 398.00^{\mathrm{a}}$ \\
\hline Samnut-22 & $1341.00 \pm 11.50^{\mathrm{bc}}$ & $4425.00 \pm 372.00^{\mathrm{abc}}$ & $3415.00 \pm 19.10^{\mathrm{a}}$ & $4192.00 \pm 368.00^{\mathrm{a}}$ \\
\hline Shar-nya & $1218.40 \pm 63.30^{\mathrm{c}}$ & $2367.00 \pm 779.00^{\mathrm{d}}$ & $889.00 \pm 16.70^{\mathrm{gh}}$ & $2833.00 \pm 402.00^{\mathrm{c}}$ \\
\hline Tisha-1 & $905.20 \pm 10.30^{\mathrm{de}}$ & $4350.00 \pm 565.00^{\mathrm{abc}}$ & $2951.00 \pm 24.70^{\mathrm{b}}$ & $3583.00 \pm 507.00^{\mathrm{abc}}$ \\
\hline$P$-Value & $\leq 0.05$ & $\leq 0.05$ & $\leq 0.05$ & 0.05 \\
\hline$C V(\%)$ & 29.13 & 21.41 & 49.53 & 26.35 \\
\hline
\end{tabular}

Mean values within each column with the same letter $(\mathrm{s})$ are not significantly different $(\mathrm{P} \leq 0.05)$ from each other according to Duncan's New Multiple Rang Test (DNMRT). CV = Coefficient of variation, DAS = Das after sowing

\section{DISCUSSIONS}

Cercospora leaf spots disease is a major constraint of groundnut production in Benue State and other parts of groundnut growing regions in Nigeria. The early leaf spot disease symptoms appearance precedes that of late leaf spot. But the two pathogens exhibited their typical distinctive disease symptoms with myriad size of leaf spots, necrotic lesions and damage. The symptoms of early leaf spot appeared brown to reddish brown in color with a yellow halo and a raised surface, (Plate 1) while the late leaf spots appeared dark brown to black spots with rough and tufted appearance but without yellow halo (plate 2). The infected groundnut leaves were seriously damaged and which as a result led to disruption of their photosynthetic activities, premature leaves defoliation and complete death of the crops. This observation was similar to those made by [15]. Also, [18] reported that leaves are site of photosynthetic activities of crop through which biomass are produced, partitioning among various parts of crop and stored for crop productivity. The reaction to early leaf spot and late leaf spot disease incidences among the groundnut cultivars assessed differed significantly in the two cropping seasons. Among the groundnut cultivars, Samnut-10, Samnut-21 did not show the symptoms of early leaf spot disease from 45 to 52 DAS in the first and second seasons; and Campalla at 45 DAS in the second season compared to other cultivars. On this trend, significantly higher incidences of late leaf spot disease was observed among Aloshi, Benue AGR, Ex-Dakar Shar-nya, and Ijiwanda from 59 to 66 DAS in the first season, Borno Brown and Borno Red were also highly infected at 66 DAS in the same season. Similarly, in the second season higher incidence of late leaf spot was observed among Shar-nya, Benue AGR, Ex-Dakar, Ijiwanda and Danbomboyo at 59 DAS, while at 66 DAS most of the groundnut cultivars exhibited significantly higher disease infection but with the exception of Smnut-10, Samnut-21, Samnut-22, Campalla and Tsha-1 which had lesser disease incidences similar to those observed at 59 DAS. The genetic trait variability among the groundnut cultivars could be responsible for the differences in the incidence of early and late leaf spot disease among the cultivars in the seasons. This informed that different groundnut cultivars can response differently to disease infection even in the same or different environmental locations. In a survey results on incidence of leaf spot diseases of groundnut conducted by [19], they reported variation in leaf spot disease incidence of $43.34 \%, 31.98 \%, 30.26 \%$ and $27.24 \%$ in 2011 and $38.12 \%, 45.36 \%, 40.25 \%$ and $35.65 \%$ in 2012 at Obi, Gboko, Guma and Makurdi agro-ecological regions of Benue State respectively. In absence of genetic disease resistance traits, crops that were not grown under protection umbrella are usually vulnerable to disease infection [19]. The findings of this study corroborate with [15] who also reported that the plant genetic physiological attributes play an important role in resistance to leaf spot disease and that high incidence of leaf spot disease infection could occur between 47 to 57 days after germination reaching highest at the pick of leaf production. Similarly, [20] also reported that variability in genetic traits exist among the groundnut cultivars they evaluated and that cultivars ICGV-SM93531, ICGV-IS-96802, ICGV-IS-96827 and ICGV-IS96808 were found to be tolerant to Cercospora leaf spot disease based on their low levels of disease incidence. Results of this study revealed that the groundnut cultivars significantly differ in their plant height and canopy spread in two cropping seasons.

There were variations and progressive increase in plant height and canopy spread among the groundnut cultivars from 55 to 75 DAS in the two cropping seasons. 


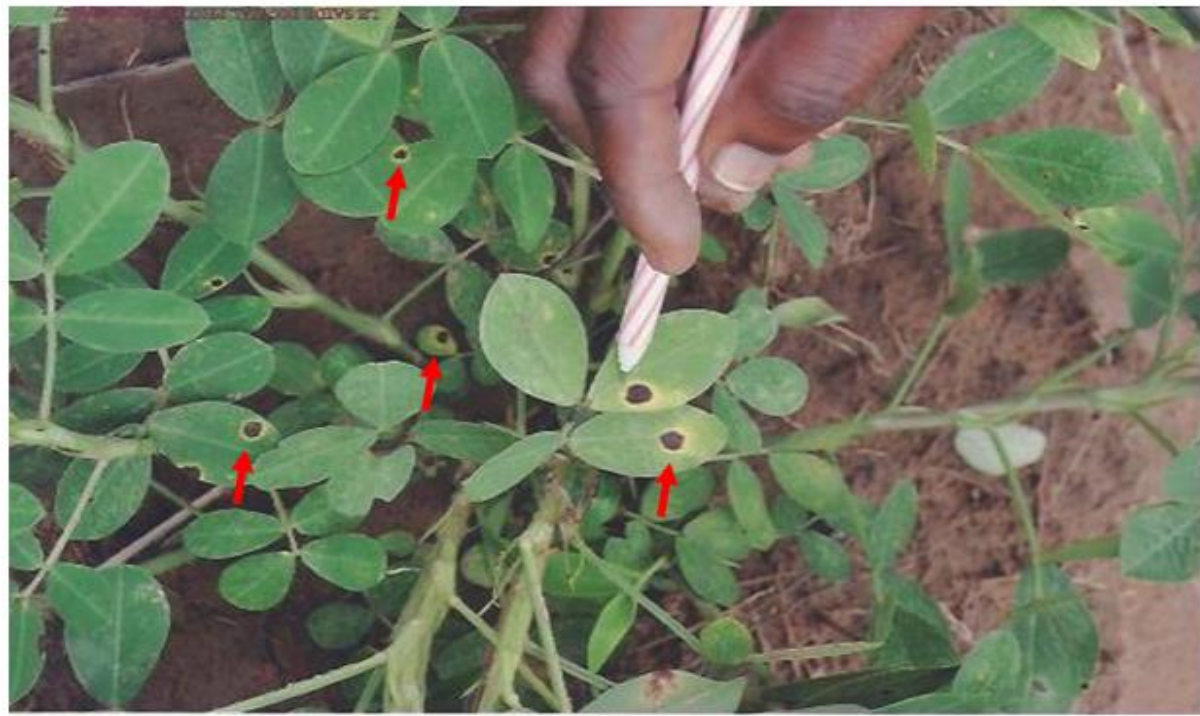

Plate 1: Early leaf spots surrounded with yellow ring halo (Cercospora arachidicola Hori)

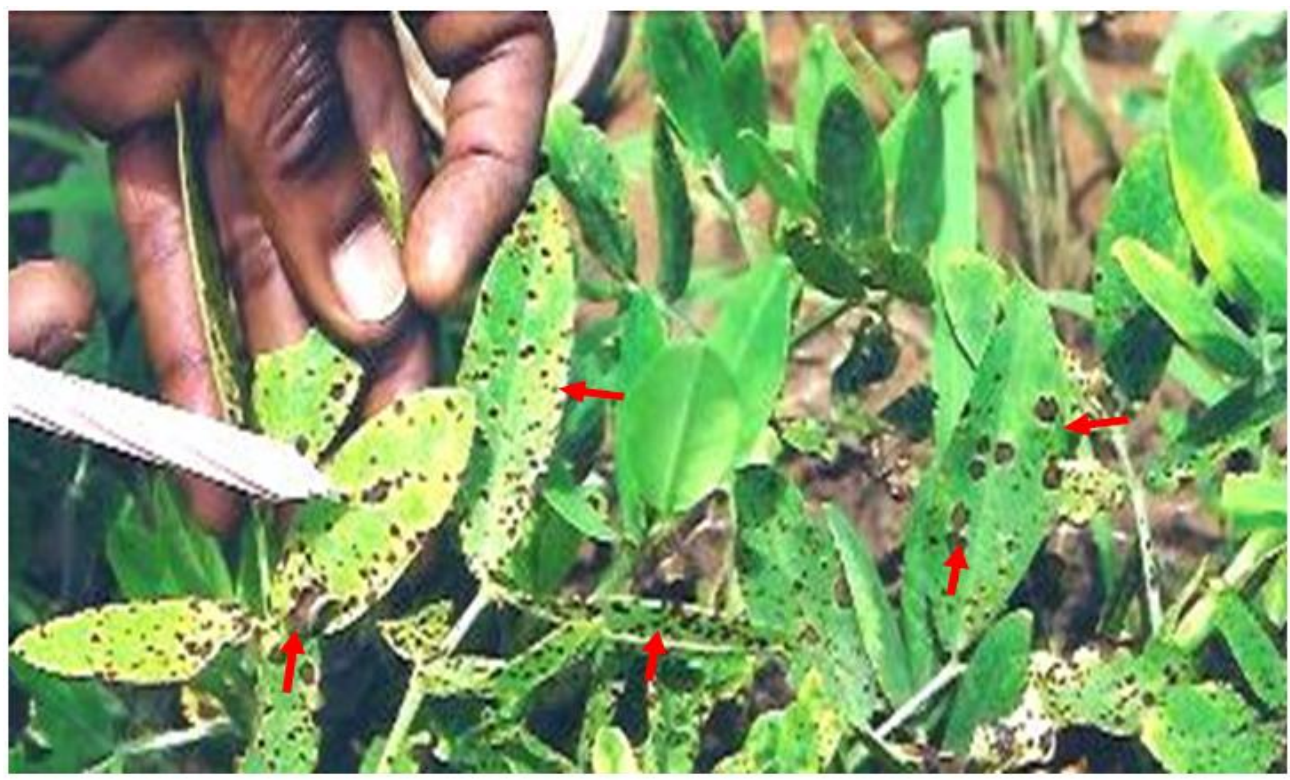

Late leaf spots (dark brown to black color) without yellow ring halo (Phaeoisariopsis personata (Berk \& Curt)

In the first season, the tallest plant height was observed among Tisha-1and Aloshi which was followed by Campalla and Samnut-22 from 55 to 75 DAS. In the second also, the tallest plant height was observed among Tisha-1, Aloshi, Benue AGR, Campalla, Danbonboyo and Ex- Dakar fromt 55 t0 75 DAS respectively. Wider canopy spread was observed among Tisha-1, Samnut-22, Campalla, Alosh and Danbomboyo from 55 to 75 DAS compared to others in both first and second seasons. The genetic make-up of these groundnut cultivars could be responsible for the variation in their agronomic performances. These findings agrees with [21] who reported wider canopy spread of $60.08 \mathrm{~cm}$ and $60.36 \mathrm{~cm}$ and lower plant height of $25.97 \mathrm{~cm}$ and $29.43 \mathrm{~cm}$ on Samnut-21 and Samnut-11 respectively; the researcher also reported 27.31, 41.56 and 47.51 number of plant branches from Samnut-23, Samnut-11 and Samnut-21 respectively.
Higher dry pod weight were obtained among Samnut-10, Samnut-21, Campalla and Samnut-22 in first season while in the second season Samnut-22, Tisha-1, Samnut-21 and Samnut-10 had higher pods yield $(\mathrm{kg} / \mathrm{ha})$ compared with other groundnut cultivars. Additionally [21], reported higher pod yields of 2285.1, 1315.1 and $1930.2 \mathrm{~kg} / \mathrm{ha}$ from Samnut-23, Samnut-21 and Samnut-11 respectively. Significantly higher haulms yield was also observed among Samnut-10, Borno Brown Samnut-21, and Samnut-22 in the two cropping seasons. Although, Campalla, Danbomboyo and Tisha-1 being local groundnut cultivars also gave a wellcome haulms yield in the two cropping seasons. In this study, it was observed that these cultivars had lower or otherwise moderate disease incidences, taller plant height and wider canopy spread, higher haulms yield and higher dry pod weight, this also corroborate with [22] who reported that the number of leaves produced 
by a plant is directly proportional to the photosynthetic produced.

\section{CONCLUSION}

In conclusion, among the groundnut cultivars assessed, Samnut-10 Samnut-21 and Samnut-22 exhibited lower disease incidence and higher pod yield characteristics. Tisha-1 Campalla and Borno Brown were moderate in their reaction to both early and late leaf spot diseases. Samnut-10 Samnut-21 and Samnut22 exhibited higher pods yield trait across the seasons Groundnut cultivars such as Borno Brown, Ex-Daker, Campalla, Samnut-21, Danbonboyo, Samnut-22, Tisha1 and Samnut-10 had higher fodder production trait and are recommended for introduction to other groundnut producing areas where groundnut haulms are highly valued for the purpose of feeding farm animals and also to serve as source of profitable income to the farmers. Lastly, Campalla, Tisha-1 and Borno Brown require further genetic improvement, they are local cultivars and there seems to be a prospect in harnessing them for the management of leaf spot disease, groundnut pod and haulm yields optimization for food sustainability for benefit of mankind.

\section{REFERENCES}

1. Ajeigbe, H. A., Waliyar, F., Echekwu, C. A., Ayuba, K., Motagi, B. N., Eniayeju, D., \& Inuwa, A. (2014). A Farmer's guide to groundnut production in Nigeria. Patancheru, 502(324), 36.

2. Weiss, E. A. (2000). Oil seed Crops. London Blackwell Science.

3. FAO. (2011). Food and Agricultural Organization of the United Nations, FAOSTAT-Database.

4. FAOSTAT. (2017). Food and Agricultural Organization of the United Nations, Statistical database.

5. Badiane, C. (2001). Senegal's trade in Groundnuts: Economic Social and Environmental implication, Senegal TED case study No. 646.

6. Kees, S. (2011). Agrometereological and groundnut production Draft by P. Vijaya Kumar reviewed by P.V. Vara Prasad revision by Kees Strighter assisted by orival do brunini.

7. Kumar, S., Jaiswal, S., Lal, A. A., Kumar, A., \& Verma, A. (2017). Influenced of natural products and bio-fungicide against tikka disease of groundnut caused by Cercospora spp. The Pharma Innovation, 6(3, Part D), 213-216.

8. Asibuo, J. Y., Akromah, R., Safo-Kantanka, O., AduDapaah, H. K., Ohemeng-Dapaah, S., \& Agyeman, A. (2008). Chemical composition of groundnut, Arachis hypogaea (L) landraces. African Journal of Biotechnology, 7(13), 2203-2208.

9. ITC. (2001). International Trade Furum-Issue 1/2001 Groundnut Facts and Plateures. Trade Forum ITC Palais des Nations. 2001; 1211 Geneva 10, Switzerland.
10. Ramesh, M. A., \& Zacharia, S. (2017). Efficacy of bio-agents and botanicals against leaf spot (Cercospora arachidicola Hori) of Groundnut (Arachis hypogaea L.). J Pharmacogn Phytochem, 6(5), 504506.

11. Ibrahim, M., Florkowski, W. J., \& Kolavalli, S. (2012). The Determination of Farmer Adoption of Improved Peanut Cultivars and Their on Farm Income. Evidence from Northern Ghana. Selected Paper Prepared for Presentation at the Agricultural and Applied Economics Association Annual Meeting, Seattle, W A, August 12-14.

12. FAOSTAT, FAOstat Statistical Data. (2017). Food and Agricultural Organization of the United Nations, Rome.

13. Thakur, S. B., Ghimire, S. K., Chaudhary, N. K., Shrestha, S. M., \& Mishra, B. (2013). Variability in groundnut (Arachis hypogaea L.) to Cercospora Leaf spot disease tolerance. Int. J. Life Sci. Biotechnol. Pharm. Res, 2, 254-262.

14. Singh, M. P., Erickson, J. E., Boote, K. J., Tillman, B. L., Jones, J. W., \& Van Bruggen, A. H. (2011). Late leaf spot effects on growth, photosynthesis, and yield in peanut cultivars of differing resistance. Agronomy journal, 103(1), 85-91.

15. Tshilenge-Lukanda, L., KKC, N., Kalonji-Mbuyi, A., \& RV, K. (2012). Epidemiology of the groundnut (Arachis hypogaea L.) leaf spot disease: genetic analysis and developmental cycles. American Journal of Plant Sciences, 2012. 3, 582-588.

16. Shokes, F. M., \& Culbreath, A. K. (1997). Early and Late Leaf spots. In; compendium of Peanut Disease, Second Edition. N. Kokalis-Burelle, N., D.M. Porter, R. Rodriguez-Kabana, D.H. Smith and P. Subrahmanyam, eds. American Phytopathology Society. St. Paul. USA. Pp. 17-20.

17. SAS, Statistical Analysis Software Package Version 9.1, SAS Institute Inc., Cary, NC, USA, 2009.

18. Asare, D. K., Frimpong, J. O., \& Ayeh, E. O. (2011). Analysis of leaf parameters of rainfed maize cultivars. American-Eurasian Journal of Agricultural and Environmental Sciences, 10(3), 338-345.

19. Richard, B. I., Ukwela, M. U., \& Avav, T. (2017). Major diseases of groundnut (Arachis hypogaea L.) in Benue State of Nigeria. Asian Journal of Advances in Agricultural Research, 4(3), 1-12.

20. Izge, A. U., Mohammed, Z. H., \& Goni, A. (2007). Levels of variability in groundnut (Arachis hypogaea L.) to cercospora leaf spot disease implication for selection. African Journal of Agricultural Research, 2(4), 182-186.

21. Mukhtar, A. A. (2011). Intensifying groundnut production in the Sudan savanna zone of Nigeria: including groundnut in the irrigated cropping systems. Pakistan journal of biological sciences: PJBS, 14(22), 1028-1031.

22. Ridge, I. (1991). Plant Physiology Hodder and Stoughton Educationtional Press. United Kingdom, $233 \mathrm{Pp}$. 